\title{
SIMULATION MODELING OF HM-NETWORKS WITH CONSIDERATION OF POSITIVE AND NEGATIVE MESSAGES
}

\author{
Victor Naumenko ${ }^{1}$, Mikhail Matalytski ${ }^{2}$ \\ ${ }^{I}$ Faculty of Mathematics and Computer Science, Grodno State University \\ Grodno, Belarus \\ ${ }^{2}$ Institute of Mathematics, Czestochowa University of Technology \\ Czestochowa, Poland \\ victornn86@gmail.com,m.matalytski@gmail.com
}

\begin{abstract}
In the article an open Queueing Network (QN) with positive and negative messages and incomes, which can be used in modeling of the behavior of viruses at the Information Systems and Networks (ISN), and also at forecasting costs taking into account virus penetration, was analyzed. Some numerical and analytical results were described on the analysis of exponential QN of the above type. It was given an algorithm of simulation modeling (SM) of HM-networks with positive and negative messages, based on the 0-point, which allows you to find incomes in such networks with arbitrary distribution of service times of positive messages. The results of the SM have been compared with the proposed analytical and numerical results. Sufficiently high accuracy of these methods was shown.
\end{abstract}

Keywords: HM-network, incomes, negative messages, simulation modeling

\section{Introduction}

It is known that the use of classical models, which take into account the characteristics of the ISN and the possible influence of various destabilizing factors, such as sudden failures from virus penetration, loss of data being processed or transmitted, does not always provide adequate results. Taking these factors into consideration, Erol Gelenbe suggested using G-networks. The idea of G-networks with negative arrivals, as well as of the relevant product form solution including non-linear traffic equations, was first published by Gelenbe in 1991, see [1]. In contrast to classical queues and $\mathrm{QN}$, the arrivals of negative customers which remove customers from a non-empty queue upon their arrival are possible in G-networks. When a negative message comes to the network system, it destroys one positive message, if any is available in the system, and reduces the number of positive messages in the system per unit. Then the negative message disappears from the network without receiving any service. For example, at ISN positive messages are programs, and negative messages are computer viruses. When a virus penetrates the computer network, it destroys or inflicts damage, infects one of the executable programs and reduces 
the number of operating programs or requests at the system by one. Then the virus disappears from the network. It is important to note that when viruses penetrate into computers in ISN, it suffers costs or losses due to the loss of information or ISN distortion. The accounting of the losses can be realized with the help of a Markov QN model with incomes (HM(Howard-Matalytski)-network), positive and negative messages. After the disappearance of the negative messages Queueing System (QS) receives again some income (profit). Such networks can be used as stochastic models for forecasting expected incomes, for example, at online payment systems on the Internet or in forecasting expenditure at ISN when the virus penetrates it or the DdoS attacks it.

In [2-4] a methodics was proposed for finding the expected incomes in systems of Markov HM-networks with a single-lines QS, as well as negative and positive messages. In this paper, we construct a simulation model of an arbitrary network and compare the results, obtained with its use, with the results obtained by other methodics. Simulation models are implemented as computer programs that reproduce events in the real system. With regard to the QN, simulation models reproduce the service processes requests in the system lines of network, message transitions from one to QS to the other, waiting messages in QS queues, etc. [5, 6]. With the help of SM computer simulation experiments it becomes possible to confirm the exact analytical methods available for finding the characteristics of the studied QN or to assess the accuracy of approximate methods.

Analytical studies are very difficult or even impossible. The advantage of SM is applicable to almost any network complexity. It should be noted, however, that it often requires a lot of time. One type of SM is a discrete-event simulation - simulation of the system at discrete moments of time when events occur, reflecting changes in the sequence of states of the system over the time. The considered systems are dynamic, which is modifiable in time. Therefore, the state of the system, the properties of the object and the number of active objects, parameters, actions and delays - is a function of time, which are constantly changing during the simulation.

\section{About the analytical modeling of HM-networks with negative messages}

Consider an open Queueing G-network with $n$ single-lines QS. At QS $S_{i}$ from outside (from the system $S_{0}$ ) is coming with a flow of positive messages with the intensity of $\lambda_{0 i}^{+}$and the Poisson flow of negative messages with the intensity of $\lambda_{0 i}^{-}, i=\overline{1, n}$. All the incoming flows of messages to the network are independent. The service time of positive messages at QS $S_{i}$ is exponentially distributed with $\mu_{i}, i=\overline{1, n}$. Each positive message is sent to the QS $S_{i}$ with the probability $p_{0 i}^{+}$, and the negative - with the probability $p_{0 i}^{-}, \sum_{i=1}^{n} p_{0 i}^{+}=\sum_{i=1}^{n} p_{0 i}^{-}=1, i=\overline{1, n}$. The posi- 
tive message was serviced at QS $S_{i}$, with the probability $p_{i j}^{+}$is sent to QS $S_{j}$ as a positive message, with the probability $p_{i j}^{-}$- as a negative message with the probability $p_{i 0}=1-\sum_{j=1}^{n}\left(p_{i j}^{+}+p_{i j}^{-}\right)$leaves from the network to the external environment (QS $\left.S_{0}\right), i, j=\overline{1, n}$. Let $k(t)=(k, t)=\left(k_{1}, k_{2}, \ldots, k_{n}, t\right)$ be $n$-vector of non-negative integers at time $t$. We denote by $\{k(t): t \geq 0\}$ the continuous-time Markov chain representing the state of the network, where $k(t)=\left(k_{1}, k_{2}, \ldots, k_{n}, t\right)$ and $k_{i}(t)$ - number of messages in queue $i$ at the time $t, i=\overline{1, n}$. A negative message coming to some network system in which there is at least one positive message immediately deletes (disrupts, removes the message from the network) and one of them thereby inflicts loss of the QS. After that, it immediately leaves the network without receiving at the QS any servicing. At each QS only positive messages may be serviced. A positive message during the transition from one QS to another QS brings the latter system some income, and income respectively of the first system is reduced by this amount. After that the negative message immediately leaves the network or is destroyed, if at the queue there are no messages, and again after servicing positive messages receives some income. The model is equivalent to the following QN with FCFS (First-come, first-served) service centers.

In [2] it is obtained a system of difference-differencial equations (DDE) to find the expected incomes systems of the network $v_{i}(k, t)$ during time $t$ on condition that at the initial time the network was in the state $k, i=\overline{1, n}$. It has the form:

$$
\begin{gathered}
\frac{d v_{i}(k, t)}{d t}=r_{i}(k)-\sum_{j=1}^{n}\left[\lambda_{0 j}^{+}+\left(\lambda_{0 j}^{-}+\mu_{j}\right) u\left(k_{j}\right)\right] v_{i}(k, t)+ \\
+\sum_{j=1}^{n}\left(\lambda_{0 j}^{+} v_{i}\left(k+I_{j}, t\right)+\left[\mu_{j} p_{j 0} u\left(k_{j}\right)+\lambda_{j j}^{-} u\left(k_{j}\right)+\mu_{j} \sum_{\substack{c=1 \\
c \neq j}}^{n} p_{j c}^{-}\left(1-u\left(k_{c}\right)\right)\right] v_{i}\left(k-I_{j}, t\right)\right]+ \\
+\lambda_{0 i}^{+} v_{i}\left(k+I_{i}, t\right)+\left[\mu_{i} p_{i 0} u\left(k_{i}\right)+\lambda_{0 i}^{-} u\left(k_{i}\right)+\mu_{i} \sum_{\substack{c=1 \\
c \neq i}}^{n} p_{i c}^{-}\left(1-u\left(k_{c}\right)\right)\right] v_{i}\left(k-I_{i}, t\right)+ \\
+\sum_{\substack{j=1 \\
j \neq i}}^{n}\left[\mu_{j} p_{j i}^{+} u\left(k_{j}\right) v_{i}\left(k+I_{i}-I_{j}, t\right)+\mu_{i} p_{i j}^{+} u\left(k_{i}\right) v_{i}\left(k-I_{i}+I_{j}, t\right)+\mu_{i} p_{i j}^{-} v_{i}\left(k-I_{i}-I_{j}, t\right)\right]+ \\
+\sum_{\substack{c, s=1 \\
c, s \neq i, c \neq s}}^{n}\left[\mu_{s} p_{s c}^{+} u\left(k_{s}\right) v_{i}\left(k+I_{c}-I_{s}, t\right)+\mu_{c} p_{c s}^{-} v_{i}\left(k-I_{c}-I_{s}, t\right)\right]+
\end{gathered}
$$




$$
\begin{gathered}
+\lambda_{0 i}^{+} r_{0 i}\left(k+I_{i}, t\right)-\left[\mu_{i} p_{i 0} u\left(k_{i}\right)+\lambda_{0 i}^{-} u\left(k_{i}\right)+\mu_{i} \sum_{\substack{c=1 \\
c \neq i}}^{n} p_{i c}^{-}\left(1-u\left(k_{c}\right)\right)\right] R_{i 0}\left(k-I_{i}, t\right)+ \\
+\sum_{\substack{j=1 \\
j \neq i}}^{n}\left[\mu_{j} p_{j i}^{+} u\left(k_{j}\right) r_{i j}\left(k+I_{i}-I_{j}, t\right)-\mu_{i} p_{i j}^{+} u\left(k_{i}\right) r_{j i}\left(k-I_{i}+I_{j}, t\right)-\right. \\
\left.-\mu_{i} p_{i j}^{-} r_{i j}\left(k-I_{i}-I_{j}, t\right)\right],
\end{gathered}
$$

where: $I_{i}$ - vector with zero components except for components with a number $i$, which equals $1, i=\overline{1, n} ; I_{0}$ - zero $n$-vector; $r_{i}(k)$ - income of system $S_{i}$ per unit time when the network is at the state $k ; r_{0 i}\left(k+I_{i}, t\right)$ - the income of system $S_{i}$, when the network makes a transition from the state $(k, t)$ to the state $\left(k+I_{i}, t+\Delta t\right)$ during time $\Delta t ;-R_{i 0}\left(k-I_{i}, t\right)$ - income of QS when the network makes a transition from the state $(k, t)$ to the state $\left(k-I_{i}, t+\Delta t\right) ; r_{i j}\left(k+I_{i}-I_{j}, t\right)$ - income of system $S_{i}$ (expense or loss of the system $S_{j}$ ), when the network makes a transition from the state $(k, t)$ to $\left(k+I_{i}-I_{j}, t+\Delta t\right)$ during time $\Delta t ;-r_{i j}\left(k-I_{i}-I_{j}, t\right)$ - income of system $S_{i}$, when the network makes a transition from the state $(k, t)$ to $\left(k-I_{i}-I_{j}, t+\Delta t\right)$ during time $\Delta t, i, j=\overline{1, n} . u(x)=\left\{\begin{array}{l}1, x>0 \\ 0, x \leq 0\end{array}\right.$. Heaviside function. If the network is able to state $k$ at moment time $t+\Delta t$, then the expected income of QS $S_{i}$ will be $r_{i}(k) \Delta t$ during time $\Delta t$ plus the expected income $v_{i}(k, t)$ that the system has received by the previous $t$ time units.

The number of equations in the system (1) is the number of states of the network, i.e. for an open network it equals $\infty$. Formally, the system of equations (1) can be reduced to a system of a countable number of inhomogeneous linear ordinary differential equations with constant coefficients.

In practice, it can be solved only in some special cases. In the case where the income from the state transition network does not depend on time, to solve the system (1) algorithms were used based on the method of successive approximations [3] and the method of multidimensional $z$-conversions [4].

In [2] an expression was also obtained for the expected incomes when incomes from the network state transitions are random variables (RV) with given average values, provided that all of the network operated in high load

$$
v_{i}(t)=v_{i 0}+\left[\lambda_{0 i}^{+} a_{0 i}-\lambda_{0 i}^{-} \bar{a}_{0 i}-\mu_{i}\left(b_{i 0} p_{i 0}+\sum_{j=1}^{n}\left(a_{i j} p_{i j}^{+}+c_{i j} p_{i j}^{-}\right)\right)+\sum_{j=1}^{n} \mu_{j} a_{j i} p_{j i}^{+}+d_{i}\right] t
$$


where $a_{0 i}, \bar{a}_{0 i}, b_{i 0}, a_{i j}, a_{j i}, c_{i j}, d_{i}$ - expectations of RV $r_{0 i}, \bar{r}_{0 i}, R_{i 0}, R_{i j}, R_{j i}, \bar{R}_{i j}$, $r_{i}$ respectively; $\xi_{i}$ - time servicing of messages at the system $S_{i}, i, j=\overline{1, n}$.

\section{Simulation modeling of HM-networks with positive and negative messages}

The simulation model of the HM-network with negative messages is a model that reproduces the process of operation of the network, aimed at obtaining a network state vector at some discrete times $t_{l}, l \geq 0[7,8]$. In simulation is set the initial state of the network at time $t_{0}$, then it constructs the trajectory of the state vector at time intervals $\left[t_{0}, t_{1}\right),\left[t_{1}, t_{2}\right), \ldots$. Moments of time $t_{l}$ correspond with moments of positive and negative messages arriving from the environment, leaving messages to the external environment (including negative), the end of the service messages at the QS and the destruction of positive messages by the negative. If, for example, a message makes a transition from system $S_{i}$ to system $S_{j}$, then $(n+i)$-th component of the state vector is reduced by 1 , a $(n+j)$-th increases by $1, i, j=\overline{1, n}$, $i \neq j$. If the positive message is destroyed at the system $S_{k}$, then $k$-th component of the state vector is reduced by 1 . In the time interval $\left[t_{l-1}, t_{l}\right)$ changes do not occur. Time points $t_{l}$ are called 0 -moments.

Thus, each 0-moment contains information about which event occurred: the arrival of a message from the outside, the end of the service message, the destruction of the positive message, from which QS the transition was done as a result of the completion of service messages, leaving the negative message of the network (without servicing) after the destruction of a positive and time occurrence of these events.

We assume that the expected incomes of network systems, depending on the state vector change as follows: in the transition from the message $i$-th QS to $j$-th, the second increases its own income by RV $r_{i j}$ with distribution function (DF) $F_{1 i}(x)$, and first reduces its income on the same RV. If the network does not make any transitions between states, each system $S_{i}$ receives income in the amount of $r_{i}$ per unit time during the period of stay in the current state of the network, where $r_{i}$ - RV with DF $F_{0 i}(x)$. In the case when at $i$-th QS destroyed a positive message, its income is reduced by RV $g_{i}$ with DF $G_{i}(x)$; if the negative message leaves the network, it does not obtain any service, the income of $i$-th QS is reduced by RV $q_{i}$, having DF $Q_{i}(x), i=\overline{1, n}$.

In modeling the initial data there will be: let network type be an open network, count of network systems $n$, matrix of transition probabilities $P$, the initial distribution of messages at the network queue systems, the number of service lines $m_{i}$ 
at network systems, $i=\overline{1, n}$, distributions of service times of positive messages, income values $r_{i}, r_{i j}, g_{i}, q_{i} i=\overline{1, n}$, and their distribution laws.

Consider each step of the algorithm for finding the expected incomes for the network with negative messages.

0 . Generation of the initial state of the network at time $t=0$ analyzes the given initial data of the simulated network. There are created a 0-points that describe the end of service some positive messages, the moments of destruction of a positive message and exit points of negative messages from the network without service after the destruction of a positive message, if any have been circulating on the network.

1. Getting a 0-moment. At this stage, the program receives information about the completion of a service of a positive message, time and number QS finish message processing, as well as information about the QS which was destroyed by a positive or negative message departure from the network. If the simulation period is over, there shall be final processing of simulation results. Otherwise, the program proceeds to step 2.

2. Processing of the current 0-moment. This performs all the activities related to the transfer message, loading other QS, the destruction of the positive message and exits from the network without service of negative messages after the destruction of the positive messages, the calculation of the new values of income of QS caused by these changes must be enumerated.

2.1. If on this step, at one of the QS with number $i, i=\overline{1, n}$, destroys a positive message, then the income of $i$-th QS decreases by the value $g_{i}$;

else if at this moment the QS was busy servicing of positive message,

then the message goes to the top of the queue of service at this QS:

- it is a calculated time priority service for this message as the difference between the length of the time interval during which the message was to be served, and the length of the time interval, during which it actually was served;

- time priority service of this message is stored.

2.2. If at this stage an event occurs associated with the departure of the QS with number $i$ negative message without servicing after the destruction of the positive message, $i=\overline{1, n}$, then:

- simulated time when the message will again be destroyed is a positive message in and out of the network according to a given distribution law;

- income $i$-th QS decreases by the value $q_{i}$;

else if in queue of $i$-th QS there are positive messages, waiting for service, it begins the service of the next message. The message will be serviced if it saved time for priority service, and is newly serviced.

2.3. If on this step, in one of the lines of QS with the number $j$ service ended, the message in accordance with the matrix of transition probabilities goes to the QS with the number $i, i, j=\overline{1, n},(i \neq j)$ and: 
if all service lines of $i$-th QS are busy,

then if puts a message in this queue of QS,

else a positive message is started serviced by a free service-line,

- time service messages are modeled according to the law of distribution with parameters corresponding to this QS;

- the end of the service time of positive message is stored; the income of $i$-th QS increases, by value $R_{i j}$, and of $j$-th decreases by the same value;

- then if it goes to the consideration of QS, finished processing of message:

if in the queue to QS with number $j$ there are any messages,

it then extracts the first positive message from the queue and begins to serve by a free line; moment of the end of the service is placed in a common event queue of the network.

3. Makes a transition to step 1 .

Simulation of the considered network at a given time interval is performed many times. The result of each experiment gets random trajectory changes in income, depending on the time. For the expected income the resulting trajectory of the network, trajectory, obtained as a result simulations, are averaged.

\section{Examples of using of simulation modeling}

Example 1. Consider an open exponential HM network with positive and negative messages, consisting of two $n=2$ QS: $S_{1}$ and $S_{2}$. The intensity of the input flow of positive and negative messages are respectively equal to: $\lambda_{01}^{+}=0.9, \lambda_{02}^{+}=3$, $\lambda_{01}^{-}=0.5, \lambda_{02}^{-}=1.2$. The intensity of message servicing at network systems are: $\mu_{1}=1, \mu_{2}=4$. Let the transition probabilities of messages be respectively equal to: $p_{12}^{+}=0.1, p_{21}^{+}=0.7$, and probabilities, that positive messages, serviced at QS $S_{i}$, sent to QS $S_{j}$, as negative messages, equal $p_{12}^{-}=0.2, p_{21}^{-}=0.2$. The probabilities of an exit of messages from the network to the external environment are: $p_{10}=0.7$, $p_{20}=0.1$. Let the income from the state transition of network equal: $r_{1}=r_{2}=4000$, $R_{10}=6000, R_{20}=4000, r_{01}=2000, r_{02}=3500, r_{12}=10000, r_{21}=10000$.

In the case when the incomes from the state transition of network is not timedependent and not dependent on network conditions, then applying the solution of the system DDE (1) algorithm based on the application of multivariate $z$-transformations described in [4], obtained the following results for expected income changes of QS $S_{2}$ (straight line), Figure 1.

With the help of SM, values were obtained for the expected incomes of QS $S_{2}$. Figure 1 shows the trajectory of behavior of an expected income of QS $S_{2}$ 
(dash line). It can be seen that the method of multivariate $z$-transformations has sufficiently high accuracy.

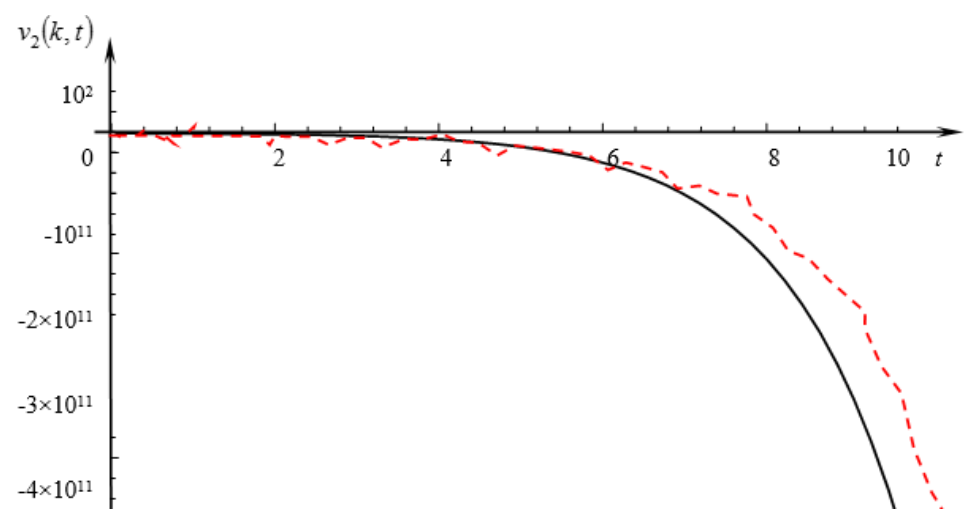

Fig. 1. Income changes of expected income for QS $S_{2}$ (straight line - method of $z$-transformations, dash line - SM)

Example 2. Consider an open exponential HM network with positive and negative messages. Let count of QS in QN be $n=4$. Consider an open exponential HM network with positive and negative messages consisting of two $\lambda_{0 i}^{+}$and $\lambda_{0 i}^{-}$be respectively equal to $\lambda_{01}^{+}=1, \lambda_{03}^{+}=2, \lambda_{01}^{-}=2, \lambda_{03}^{-}=5$, others are equal to zero. The intensity of message servicing at network systems $\mu_{i}$ equals $\mu_{1}=\mu_{2}=\mu_{3}=6$, $\mu_{4}=0.5$. Let probabilities $p_{i j}^{+}$be respectively equal to $p_{12}^{+}=1 / 8, p_{13}^{+}=1 / 8$, $p_{21}^{+}=p_{24}^{+}=1 / 10, \quad p_{31}^{+}=p_{34}^{+}=1 / 10, \quad p_{42}^{+}=p_{43}^{+}=1 / 8$, others are equal to zero. The probability that the positive messages serviced at QS $S_{i}$, send to QS $S_{j}$, as a negative message, equal to $p_{12}^{-}=1 / 9, p_{13}^{-}=1 / 9, p_{21}^{-}=1 / 11, p_{24}^{-}=1 / 11$, $p_{31}^{-}=1 / 11, p_{34}^{-}=1 / 11, p_{42}^{-}=p_{43}^{-}=1 / 9$, the others are equal to zero. The probabilities of exit of messages from the network to the external environment are: $p_{20}=34 / 55, p_{4,0}=19 / 36, p_{10}=p_{30}=0$. The incomes of $S_{i}$ are respectively equal to $r_{i}=2500, i=\overline{1, n} ; r_{i j}(k)=1000 \sin a$, where $a$ - number of renumbered state $k$, $i, j=\overline{1, n}$. Let incomes be $r_{0 i}(k)=2000 \sin a, R_{i 0}(k)=1000 \sin a, i=\overline{1, n}$. Because the number of states in our network is infinite, then the value $a$ at each time moment $t$ changes. Let, for example, at moment time $t=1$, there be 7 messages in the network, consequently, the number of states at this time be equal to 120 . They are numbered from 1 to 120 , i.e. at time $t=1 \quad a=\overline{1,120}$. In this case, we assume that the state $(0,0,0,0)$ be a state of number one.

Figure 2 shows the income change of QS $S_{2}$ (straight line) depending on the time. Here, for the solution of system (1), is used the method of successive approximations with accuracy of $\varepsilon=10^{-6}$ [3], when incomes from transitions between network states 
depend on network states and don't depend on time. The initial state of network was $(0,0,0,0)$. The income at initial moment time equals $V(k, 0)=0$. Averaging of the trajectory obtained as a result of simulations for this example have also received at Figure 2 a chart of income of QS $S_{2}$ (dash line), which shows that the approximate method of successive approximations has a sufficiently high accuracy.

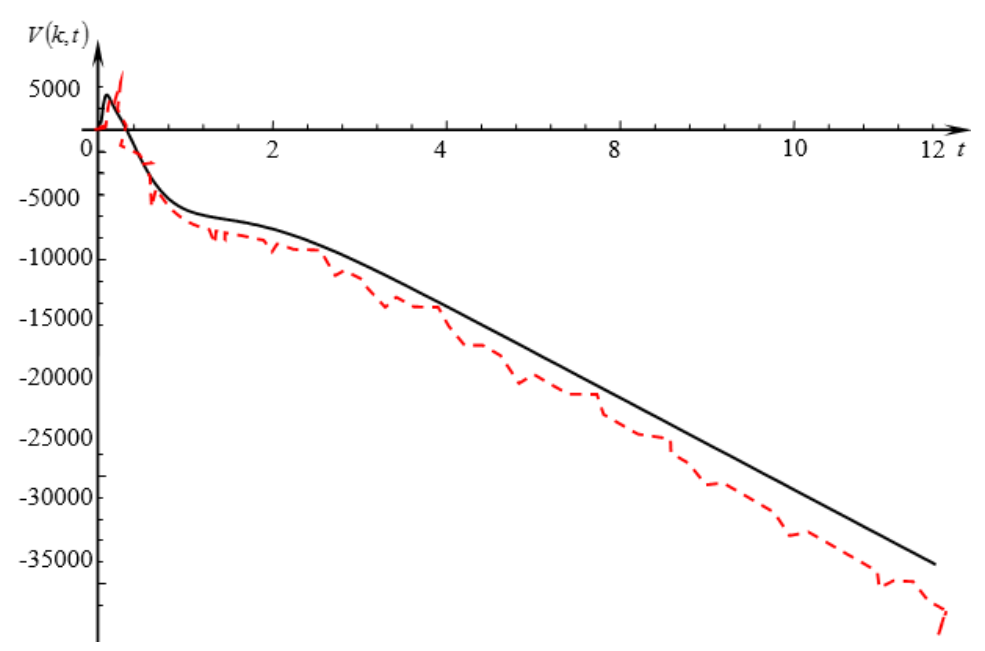

Fig. 2. Income changes of system $S_{2}$ in numerical calculations and SM (straight line - method of successive approximations, dash line - SM)

Example 3. Consider now the HM-network with positive and negative messages and random incomes. Let the number of the QS equal $n=10$. The intensity of the input flows of positive and negative messages $\lambda_{0 i}^{+}$and $\lambda_{0 i}^{-}$are respectively equal to $\lambda_{01}^{+}=1, \lambda_{04}^{+}=3, \lambda_{07}^{+}=2, \lambda_{01}^{-}=2, \lambda_{04}^{-}=3, \lambda_{07}^{-}=2$, the others are zero. The intensity of service messages $\mu_{i}$ equals $\mu_{1}=\mu_{2}=\mu_{3}=1, \mu_{4}=2, \mu_{5}=2, \mu_{6}=4, \mu_{7}=2$, $\mu_{8}=10, \mu_{9}=6, \mu_{10}=7$. Let the probabilities $p_{i j}^{+}$be respectively equal to

$$
\begin{gathered}
p_{12}^{+}=p_{13}^{+}=p_{42}^{+}=p_{45}^{+}=p_{48}^{+}=p_{52}^{+}=p_{54}^{+}=p_{56}^{+}=p_{58}^{+}= \\
=p_{63}^{+}=p_{65}^{+}=p_{67}^{+}=p_{69}^{+}=p_{73}^{+}=p_{76}^{+}=p_{79}^{+}= \\
=p_{84}^{+}=p_{85}^{+}=p_{89}^{+}=p_{8,10}^{+}=p_{96}^{+}=p_{97}^{+}=p_{98}^{+}=p_{9,10}^{+}=\frac{1}{8}, \\
p_{21}^{+}=p_{23}^{+}=p_{24}^{+}=p_{25}^{+}=p_{21}^{+}=p_{31}^{+}=p_{32}^{+}=p_{36}^{+}=p_{37}^{+}=0.1, \\
p_{10,8}^{+}=p_{10,9}^{+}=0.2,
\end{gathered}
$$

the others are equal to zero. The probabilities that the positive messages that were served at QS $S_{i}$ are sent to QS $S_{j}$ as negative messages are equal to 


$$
\begin{gathered}
p_{12}^{-}=p_{13}^{-}=p_{42}^{-}=p_{45}^{-}=p_{48}^{-}=p_{52}^{-}=p_{54}^{-}=p_{56}^{-}=p_{58}^{-}=p_{63}^{-}=p_{65}^{-}=p_{67}^{-}= \\
=p_{69}^{-}=p_{69}^{-}=p_{73}^{-}=p_{73}^{-}=p_{79}^{-}=p_{84}^{-}=p_{85}^{-}=p_{89}^{-}=p_{8,10}^{-}=p_{96}^{-}=p_{97}^{-}=p_{98}^{-}=p_{9,10}^{-}=\frac{1}{9}, \\
p_{21}^{-}=p_{23}^{-}=p_{24}^{-}=p_{25}^{-}=p_{31}^{-}=p_{32}^{-}=p_{36}^{-}=p_{37}^{-}=p_{10,8}^{-}=p_{10,9}^{-}=\frac{1}{11}, \\
p_{10,8}^{-}=p_{10,9}^{-}=\frac{1}{6},
\end{gathered}
$$

the others are equal to zero. The probability of exit messages from the network to the external environment $p_{40}=p_{70}=\frac{7}{24}, p_{10,0}=\frac{4}{15}$. The expectations are respectively equal to

$$
\begin{gathered}
a_{01}=a_{05}=a_{06}=a_{010}=\bar{a}_{03}=10000, a_{02}=20000, a_{03}=a_{08}=30000, \\
a_{04}=a_{09}=\bar{a}_{01}=\bar{a}_{05}=50000, a_{07}=40000, \bar{a}_{02}=60000, \bar{a}_{04}=25000, \\
\bar{a}_{06}=7000, \bar{a}_{07}=3000, \bar{a}_{08}=2500, \bar{a}_{09}=1200, \bar{a}_{010}=9000, \\
b_{10}=b_{20}=b_{50}=b_{60}=b_{70}=b_{10,0}=1000, b_{40}=b_{90}=5000, \\
b_{30}=b_{80}=3000, b_{40}=b_{90}=5000, \\
c_{12}=c_{31}=c_{32}=c_{36}=c_{37}=1000, \\
c_{21}=c_{23}=c_{24}=c_{25}=c_{45}=c_{45}=c_{48}=c_{84}=c_{85}=c_{89}=c_{8,10}=2000, \\
c_{96}=c_{97}=c_{98}=c_{9,10}=3000, c_{31}=c_{52}=c_{54}=c_{56}=c_{58}=1500, \\
c_{63}=c_{65}=c_{67}=c_{59}=1700, c_{73}=c_{76}=c_{79}=2300, c_{10,8}=c_{10,9}=1300, \\
d_{1}=100, d_{2}=200, d_{1}=100, d_{3}=300, d_{4}=120, \\
d_{5}=200, d_{6}=100, d_{7}=800, d_{8}=100, d_{9}=120, \\
a_{13}=3000, a_{13}=1000, a_{13}=1000, a_{21}=2500, a_{23}=100, a_{24}=200, \\
a_{25}=2000, a_{31}=1000, a_{32}=10000, a_{36}=450, a_{37}=5000, a_{42}=100, \\
a_{45}=100, a_{37}=200, a_{52}=1000, a_{54}=2000, a_{56}=3000, a_{58}=4000, \\
a_{63}=1000, a_{65}=1500, a_{67}=3000, a_{69}=2000, a_{73}=100, a_{76}=150, \\
a_{79}=300, a_{84}=500, a_{85}=700, a_{89}=600, a_{8,10}=200, \\
a_{96}=700, a_{97}=1000, a_{98}=900, a_{9,10}=600, a_{96}=3000, a_{96}=5500,
\end{gathered}
$$

others are equal to zero. Consider a time interval of length $24 \mathrm{~h}, t \in[0, T], T=24$. In [2] one of the methods has been described by finding expressions for the expected incomes. For example, the expression for the expected income of system $S_{1}$ considering (2) has the form $v_{1}(t)=-800 t$. 


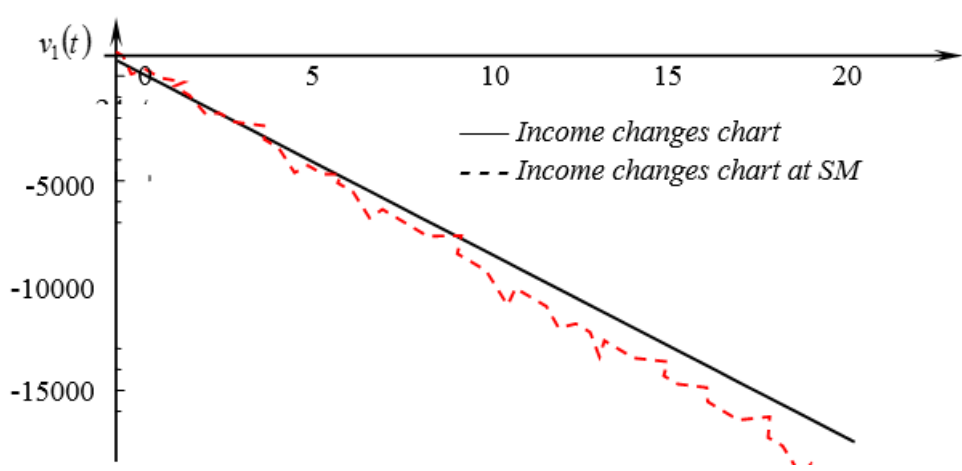

Fig. 3. Income changes of QS $S_{1}$

Figure 3 shows the income changes at system $S_{3}$ for the HM-network with negative messages and the result SM of its network. These results confirm a sufficiently high accuracy of the formula (2).

\section{Conclusions}

In the present study a SM of HM-network with positive and negative messages has been made, with the help of which we can find the expected incomes in the systems with arbitrary laws of distribution of service times of messages in its QS. A series of simulation experiments was performed, during which it became clear that the expected incomes were quite similar with the same incomes obtained by numerical and approximate analytical methods. By examining the results of these experiments, it can also be noted that with the help of the previously proposed methods we can find the expected incomes with an infinite number of states of networks in a reasonable CPU time. Once again, we note that the results obtained allow one to simulate the behavior of viruses at the ISN, spending forecast costs of ISN at DDoS-attacks or control the load at ISN.

\section{References}

[1] Gelenbe E., Product form queueing networks with negative and positive customers, Journal of Applied Probability 1991, 28, 656-663.

[2] Naumenko V., Matalytski M., Analysis of Markov networks with incomes, positive and negative messages, Informatics 2014, 1, 5-14.

[3] Naumenko V., Income analysis at Markov G-networks by successive approximation method, Vestnik of GrSU 2014, 2, 1, 143-157.

[4] Matalytski M., Naumenko V., Application of a z-transforms method for investigation of Markov G-networks, J. Appl. Math. Comput. Mech. 2014, 13(1), 61-73. 
[5] Dai J.G., Simulation studies of multiclass queueing networks, IEEE Transactions 1997, 29(3), 213-219.

[6] Pankov A., Matalytski M., Computer simulation of incomes in queueing networks and its application, Computer Science 2005, 5(9), 29-36.

[7] Astakhov A.M., Simulation modeling of systems with negative customers and their application to the analysis of OBS-networks, Vestnik of GrSU 2008, 2, 3, 138-144.

[8] Statkevich C.E., Simulation modeling HM-networks with unreliable service, Vestnik of GrSU 2012, 2, 2, 129-134.

[9] Naumenko V., Matalytski M., Analysis of Markov net with incomes, positive and negative customers, Informatics 2014, 1, 5-14. 